\title{
On the Question of Asymptotic Integration of Singularly Perturbed Fractional-Order Problems
}

\author{
Burkhan Kalimbetov \\ Department of Mathematics Akhmet Yasawi University \\ Turkestan, Kazakhstan, \\ E-mail: burkhan.kalimbetov [AT] ayu.edu.kz
}

\begin{abstract}
In this paper we consider an initial problem for systems of differential equations of fractional order with a small parameter for the derivative. Regularization problem is produced, and algorithm for normal and unique solubility of general iterative systems of differential equations with partial derivatives is given.
\end{abstract}

Keywords--- matrix-function, vector-function, differential equation of fractional order, regularization, asymptotic, iterative problems, normal and unique solvability.

\section{INTRODUCTION}

We consider the following singularly perturbed problem:

$$
L_{\varepsilon} y(t, \varepsilon) \equiv \varepsilon y^{(1 / 2)}-A(t) y=h(t), \quad y(0, \varepsilon)=y^{0}, \quad t \in[0, T]
$$

where $y \equiv\left\{y_{1}, y_{2}\right\}$ unknown vector-function, $h(t) \equiv\left\{h_{1}, h_{2}\right\}$ known vector-function, $A(t)-2 \times 2$ matrix-function, $y^{0}=\left\{y_{1}^{0}, y_{1}^{0}\right\}$ known constant vector, $\varepsilon>0$ small parameter. It is required to construct a regularized asymptotic of a solution $[1,2]$ of the problem (1) at for $\varepsilon \rightarrow+0$.

According to the definition of a fractional order derivative $[3,4,5]$, we write the problem (1) in the following form:

$$
L_{\varepsilon} y(t, \varepsilon) \equiv \varepsilon \sqrt{t} \cdot y^{\prime}-A(t) y=h(t), \quad y(0, \varepsilon)=y^{0}, \quad t \in[0, T]
$$

We will consider the problem (2) under the following assumptions:

1) $A(t), h(t) \in C^{\infty}\left([0, T], \square^{2}\right)$,

2) the spectrum $\left\{\lambda_{j}(t)\right\} \equiv \sigma(A(t))$ of the matrix function $A(t)$ satisfies the requirements:

ai) $\lambda_{j}(t) \neq 0 \quad \forall t \in[0, T], \quad j=\overline{1,2}$;

b) $\lambda_{i}(t) \neq \lambda_{j}(t) \quad \forall t \in[0, T], \quad i \neq j, i, j=\overline{1,2}$;

c) $\operatorname{Re} \lambda_{j}(t) \leq 0 \forall t \in[0, T], j=\overline{1,2}$.

\section{REGULARIZATION OF THE PROBLEM}

We introduce regularizing variables [6]:

$$
\tau_{j}=\frac{1}{\varepsilon} \int_{0}^{t} \frac{\lambda_{j}(s)}{\sqrt{s}} d s \equiv \varphi_{j}(t, \varepsilon), j=1,2
$$

and instead of the problem (2), we will consider «extended» problem

$$
L_{\varepsilon} \tilde{y}(t, \tau, \varepsilon) \equiv \varepsilon \sqrt{t} \frac{\partial \tilde{y}}{\partial t}+\sum_{j=1}^{2} \lambda_{j}(t) \frac{\partial \tilde{y}}{\partial \tau_{j}}-A(t) \tilde{y}=h(t), \quad \tilde{y}(0,0, \varepsilon)=y^{0} .
$$

Relations of the problem (3) with the problem (2) is that if $\tilde{y}(t, \tau, \varepsilon)$ is a solution of the problem (3), then contraction of the solution

$$
\tilde{y}\left(t, \varphi_{1}(t, \varepsilon), \varphi_{2}(t, \varepsilon), \varepsilon\right) \equiv y(t, \varepsilon)
$$

when $\left.\tau_{1}=\varphi_{1}(t, \varepsilon), \tau_{2}=\varphi_{2}(t, \varepsilon), \varepsilon\right)$ will be exact solution of the problem (2).

Defining a solution of the system (34) in the form of series:

$$
\tilde{y}(t, \tau, \varepsilon)=\sum_{k=0}^{\infty} \varepsilon^{k} y_{k}(t, \tau), y_{k}(t, \tau) \in C^{\infty}\left([0, T], C^{2}\right)
$$


we obtain the following iteration problems:

$$
\begin{gathered}
L y_{0}(t, \varepsilon) \equiv \sum_{j=1}^{2} \lambda_{j}(t) \frac{\partial y_{0}}{\partial \tau_{j}}-A(t) y_{0}=h(t), \quad y_{0}(0,0)=y^{0} ; \\
L y_{1}(t, \varepsilon)=-\sqrt{t} \frac{\partial y_{0}}{\partial t}, \quad y_{1}(0,0)=0 \quad ; \\
L y_{k}(t, \varepsilon)=-\frac{\partial y_{k-1}}{\partial t}, \quad y_{k}(0,0)=0, \quad k \geq 1 .
\end{gathered}
$$

\section{SOLVABILITY OF ITERATION PROBLEMS}

Solution of each of the iteration problems (5)-(7) will be defined in the space $U$ of functions of the form:

$$
U=\left\{y(t, u): y=y_{0}(t)+\sum_{j=1}^{2} y_{j}(t) e^{\tau_{j}}, \quad y_{j}(t) \in C^{\infty}\left([0, T], C^{2}\right)\right\} .
$$

Each of the iteration problems (5)-(7) has the following form:

$$
L y(t, \varepsilon) \equiv \sum_{j=1}^{2} \lambda_{j}(t) \frac{\partial y_{0}}{\partial \tau_{j}}-A(t) y_{0}=h(t, \tau)
$$

where $h(t, \tau) \in U$ corresponding right hand side.

The following proposition takes place.

Theorem 1. Let $h(t, \tau) \in U$ and conditions 1) and 2a), 2b) hold. Then, for solvability of the equation (9) in space $U$, it is necessary and sufficient that the following conditions hold:

$$
<h(t, \tau), d_{j}(t)>\equiv 0, \quad j=1,2, \quad \forall t \in[0, T]
$$

where $d_{j}(t)$ eigenfunctions of the matrix of functions $A^{*}(t)$, corresponding to eigenvalues $\bar{\lambda}_{j}(t), j=1,2$.

Proof. Defining a solution $y(t, \tau)$ of the system (9) as an element (8) of the space $U$, we get the following systems for the coefficients $y_{j}(t), j=0,1,2$, of the sum (8):

$$
\begin{aligned}
& {\left[\lambda_{k}(t) I-A(t)\right] y_{k}(t)=h_{k}(t), \quad k=1,2,} \\
& -A(t) y_{0}(t)=h_{0}(t), \quad(I \equiv \operatorname{diag}(1,1)) .
\end{aligned}
$$

The system (12), due to $\operatorname{det} A(t) \neq 0$, has a unique solution $y_{0}(t)=-A^{-1}(t) h_{0}(t)$. The system (11) is solvable in $C^{\infty}[0, T]$ if and only if the condition $\left\langle h_{k}(t), d_{k}(t)>\equiv 0, k=1,2, \forall t \in[0, T]\right.$, holds, that coincides with the condition (10). Theorem 1 is proved.

Remark. If the conditions (10) hold, system (9) has a solution that can be represented as

$$
y(t, \tau)=\sum_{k=1}^{2}\left[\alpha_{k}(t) c_{k}(t)+\sum_{\substack{s \neq k \\ s=1}}^{2} \frac{\left(h_{k}(t), d_{s}(t)\right)}{\lambda_{k}(t)-\lambda_{s}(t)} c_{s}(t)\right] e^{\tau_{k}}-A^{-1}(t) h_{0}(t)
$$

where $\alpha_{k}(t) \in C^{\infty}[0, T], k=1,2$, arbitrary scalar functions.

The following theorem establishes conditions under which the solution (13) of system (9) is uniquely defined in the class $U$.

Theorem 2. Let 1), 2a), 2b) hold and $h(t, \tau) \in U$ of the system (9) satisfy conditions (10). Then the system (10) with additional conditions:

$$
\begin{gathered}
y(0,0)=y^{0}, \\
<-\sqrt{t} \frac{\partial y(t, \tau)}{\partial t}, d_{j}(t)>\equiv 0, \quad j=1,2, \forall t \in[0, T]
\end{gathered}
$$

where $y^{0} \in C^{n}$ known constants, is uniquely solvable in the space $U$.

Proof. Since conditions of Theorem 1 hold, the system (9) has a solution in the space $U$ in the form (13), where functions $\alpha_{k}(t), k=1,2$, have not yet been found. To calculate them, we will use additional conditions (14) and (15).

We subject (13) to the initial condition (14), we get the system: 


$$
\sum_{k=1}^{2}\left[\alpha_{k}(0) c_{k}(0)+\sum_{s \neq k, s=1}^{2} \frac{\left(h_{k}(0), d_{s}(0)\right)}{\lambda_{k}(0)-\lambda_{s}(0)} c_{s}(0)\right]-A^{-1}(0) h_{0}(0)=y^{0} .
$$

Multiplying scalarly both sides of this equality by $d_{k}(0)$ and taking into account biorthogonality of the systems $\left\{c_{k}(t)\right\}$ and $\left\{d_{k}(t)\right\}$ we uniquely find initial values $\alpha_{k}(0)=\alpha_{k}^{0}$ for the functions $\alpha_{k}(t), k=1,2$.

We subject now the function (13) to the condition (15). First calculate $\frac{\partial y(t, \tau)}{\partial t}$ :

$$
\sum_{k=1}^{2}\left\{\left(\alpha_{k} c_{k}^{\prime}+\alpha_{k}^{\prime} c_{k}\right)+\left[\sum_{s \neq k, s=1}^{2} \frac{\left(h_{k}, d_{s}\right)^{\prime}\left(\lambda_{k}-\lambda_{s}\right)-\left(h_{k}, d_{s}\right)\left(\lambda_{k}-\lambda_{s}\right)^{\prime}}{\lambda_{k}-\lambda_{s}} c_{s}+\frac{\left(h_{k}, d_{s}\right)}{\lambda_{k}-\lambda_{s}} c_{s}^{\prime}\right]\right\} e^{\tau_{k}}-\left(A^{-1} h_{0}\right)^{\prime} .
$$

Conditions (15) lead to the equations:

$$
-\sqrt{t}\left[\alpha_{k}^{\prime}+\left(c_{k}^{\prime}, d_{k}\right) \alpha_{k}+\sum_{\substack{s \neq k \\ s=1}}^{2} \frac{\left(h_{k}, d_{s}\right)}{\lambda_{k}-\lambda_{s}}\left(c_{k}^{\prime}, d_{k}\right)-\left(\left(A^{-1} h_{0}\right)^{\prime}, d_{k}\right)\right]=0, k=1,2 .
$$

which together with the initial conditions $\alpha_{k}(0)=\alpha_{k}^{0}$, found earlier, allow us to uniquely find the functions $\alpha_{k}(t), k=1,2$. Theorem 2 is proved.

Thus, the solution (13) of the problem in the space $U$ is found unambiguously. Solutions of the next iteration problems (6),(7),.. are found similarly in the space. Doing it, we construct the series (4). Denote by $\left.y_{\varepsilon N}(t) \equiv \sum_{k=1}^{N} \varepsilon^{k} y_{k}(t, \tau)\right|_{\tau=\varphi(t, \varepsilon)}$ constriction of the $N-$ th partial sum of the series at $\tau=\varphi(t, \varepsilon)$. The following proposition takes place.

Theorem 3 (on formal asymptotic solution of the problem (2)). Let conditions 1) - 2) be fulfilled. Then the partial sum $y_{\varepsilon N}(t)$ satisfies the problem (2) up to $O\left(\varepsilon^{N+1}\right)(\varepsilon \rightarrow+0)$, i.e.

$$
\varepsilon \sqrt{t} \frac{d y_{\varepsilon N}(t)}{d t} \equiv A(t) y_{\varepsilon N}(t)+h(t)+\varepsilon^{N+1} R_{N}(t, \varepsilon), y_{\varepsilon N}(0)=y^{0}, \forall t \in[0, T]
$$

where $\left\|R_{N}(t, \varepsilon)\right\|_{C[0, T]} \leq \bar{R}_{N}$ at all $t \in[0, T]$ and $\varepsilon>0$.

Proof. We put solutions $y_{0}(t, \tau), \ldots, y_{N}(t, \tau)$ into the systems (5),(6),(7),...respectively. We multiply the resulting identities by $1, \varepsilon, \ldots, \varepsilon^{N}$ respectively, and summing up them, we will have identities:

$$
\begin{gathered}
L\left(\sum_{k=0}^{N} \varepsilon^{k} y_{k}(t, \tau)\right) \equiv h(t)-\varepsilon \sum_{k=0}^{N-1} \varepsilon^{k} \frac{\partial y_{k}(t, \tau)}{\partial t} \Leftrightarrow \\
\Leftrightarrow L\left(\sum_{k=0}^{N} \varepsilon^{k} y_{k}(t, \tau)\right)+\varepsilon \sum_{k=0}^{N-1} \varepsilon^{k} \frac{\partial y_{k}(t, \tau)}{\partial t} \equiv h(t)+\varepsilon^{N+1} \frac{\partial y_{N}(t, \tau)}{\partial t} .
\end{gathered}
$$

Denoting by $S_{N}(t, \varepsilon) \quad N$-th partial sum of the series (4), we write this identity in the form:

$$
\begin{gathered}
\varepsilon \frac{\partial S_{N}(t, \tau, \varepsilon)}{\partial t}+L S_{N}(t, \tau, \varepsilon) \equiv h(t)+\varepsilon^{N+1} \frac{\partial y_{N}(t, \tau, \varepsilon)}{\partial t} \Leftrightarrow \\
\Leftrightarrow \varepsilon \frac{\partial S_{N}(t, \tau, \varepsilon)}{\partial t}+\sum_{j=1}^{2} \lambda_{j}(t) \frac{\partial S_{N}(t, \tau, \varepsilon)}{\partial \tau_{j}} \equiv A(t) S_{N}(t, \tau, \varepsilon)+h(t)+\varepsilon^{N+1} \frac{\partial y_{N}(t, \tau, \varepsilon)}{\partial t} .
\end{gathered}
$$

This identity is true at all $(t, \tau, \varepsilon) \in[0, T] \times \square^{2} \times\{\varepsilon>0\}$, thus, it, particularly, is true at $\tau=\varphi(t, \varepsilon)$. However at $\tau=\varphi(t, \varepsilon)$ the left hand side of this identity coincides with full derivative with respect to $t$ of the function $y_{N}(t) \equiv S_{N}(t, \varphi(t, \varepsilon), \varepsilon)$, therefore, we will have:

$$
\varepsilon \sqrt{t} \frac{d y_{\varepsilon N}(t)}{d t} \equiv A(t) y_{\varepsilon N}(t)+h(t)+\varepsilon^{N+1} \frac{\partial y_{N}(t, \varphi(t, \varepsilon))}{\partial t} .
$$

Vector function is $y_{N}(t, \tau) \in U$, hence it is represented as $y_{N}(t, \tau)=\sum_{j=1}^{2} y_{j}^{(N)}(t) e^{\tau_{j}}+y_{0}^{(N)}(t)$ and thus, 


$$
\left\|\frac{\partial y_{N}(t, \varphi(t, \varepsilon))}{\partial t}\right\|_{C[0, T]} \leq \sum_{j=1}^{2}\left\|\dot{y}_{j}^{(N)}(t)\right\|_{C[0, T]} \max _{t \in[0, T]} e^{\frac{1}{\int_{\varepsilon}^{\prime}} \operatorname{l}_{0} \lambda_{j}(\theta) d \theta}+\left\|\dot{y}_{j}^{(N)}(t)\right\|_{C[0, T]} \leq \sum_{j=1}^{2}\left\|\dot{y}_{j}^{(N)}(t)\right\|=\bar{R}_{N} .
$$

Here $\operatorname{Re} \lambda_{j}(t) \leq 0(\forall t \in[0, T])$, and then $\left.\exp \left\{\frac{1}{\varepsilon} \int_{0}^{t} \operatorname{Re} \lambda_{j}(\theta) d \theta\right\} \leq 1(\forall t \in[0, T]), \forall \varepsilon>0, j=1,2\right)$. It remains to be noted that the function $y_{\varepsilon N}(t)$ satisfies the initial condition $y_{\varepsilon N}(0)=y^{0}$, since $y_{0}(0,0)=y^{0}$ and all $y_{j}(0,0)=0$ whenever $j>1$. Theorem 3 is proved.

Theorem 3 shows, that the series (4), take non constriction $\tau=\varphi(t, \varepsilon)$, is a formal asymptotic solution of the problem (2). We show that in fact it converges asymptotically (as $\varepsilon \rightarrow+0$ ) to an exact solution $y(t, \varepsilon)$ of this problem (uniformly with respect to $t \in[0, T]$ ). Let us now prove the following main proposition.

Theorem 4 (on estimation of remainder member). Let conditions 1) - 2) hold. Then the series (4) taken on constriction $\tau=\varphi(t, \varepsilon)$, is uniform with respect to $t \in[0, T]$ asymptotic decomposition as $\varepsilon \rightarrow+0$ ) of an exact solution $y(t, \varepsilon)$ of the problem (2). Moreover, for any of its partial sums $y_{\varepsilon N}(t)$ the following estimate is valid

$$
\left\|y(t, \varepsilon)-y_{\varepsilon N}(t)\right\|_{C[0, T]} \leq C_{N} \varepsilon^{N+1}(N=0,1,2, \ldots)
$$

where the constant $C_{N}>0$ does not depend on $\varepsilon$ when $\varepsilon>0$.

Proof. Due to Theorem 3 the partial sum $y_{\varepsilon N}(t)$ satisfies the problem (16) and thus, remainder member $\Delta_{N}(t, \varepsilon) \equiv y(t, \varepsilon)-y_{\varepsilon N}(t)$ satisfies the following problem:

$$
\varepsilon \frac{d \Delta_{N}(t, \varepsilon)}{d t}=A(t) \Delta_{N}(t, \varepsilon)-\varepsilon^{N+1} R_{N}(t, \varepsilon), \Delta_{N}(0, \varepsilon)=0 .
$$

Using the normal fundamental matrix of solutions $Y(t, s, \varepsilon)$, we find

$$
\Delta_{N}(t, \varepsilon)=-\int_{0}^{t} Y(t, s, \varepsilon) R_{N}(t, \varepsilon) d s .
$$

Therefore, we get the estimation:

$$
\left\|\Delta_{N}(t, \varepsilon)\right\|_{C[0, T]} \leq k_{0} \bar{R}_{N} T \varepsilon^{N}
$$

that is validate any $N=0,1,2, \ldots$, and any $\varepsilon>0$ and thus, for the partial sum $y_{\varepsilon, N+1}(t) \equiv y_{\varepsilon N}(t)+\varepsilon^{N+1} y_{N+1}(t, \varphi(t, \varepsilon))$ the following estimation holds:

$$
\left\|y(t, \varepsilon)-y_{\varepsilon, N+1}(t)\right\|_{C[0, T]} \equiv\left\|\left(y(t, \varepsilon)-y_{\varepsilon N}(t)\right)-\varepsilon^{N+1} y_{N+1}(t, \varphi(t, \varepsilon))\right\|_{C[0, T]} \leq k_{0} \bar{R}_{N+1} T \varepsilon^{N+1} .
$$

Using the inequality $\|a-b\| \geq\|a\|-\|b\|$, we have

$$
\left\|y(t, \varepsilon)-y_{\varepsilon N}(t)\right\|_{C[0, T]}-\varepsilon^{N+1}\left\|y_{N+1}(t, \varphi(t, \varepsilon))\right\|_{C[0, T} \leq k_{0} \bar{R}_{N+1} T \varepsilon^{N+1}
$$

that yields the simple estimation:

$$
\left\|y(t, \varepsilon)-y_{\varepsilon N}(t)\right\|_{C[0, T]} \leq\left(k_{0} \bar{R}_{N+1} T+\bar{Q}_{N+1}\right) \varepsilon^{N+1} \equiv C_{N+1} \varepsilon^{N+1}
$$

where

$$
\left\|y_{N+1}(t, \varphi(t, \varepsilon))\right\|_{C[0, T} \equiv\left\|\sum_{j=1}^{2} y_{j}^{(N+1)}(t) e^{\varphi_{j}(t, \varepsilon)}+y_{0}^{(N+1)}(t)\right\|_{C[0, T]} \leq \sum_{j=1}^{2}\left\|y_{j}^{(N+1)}(t)\right\|_{C[0, T]} \equiv \bar{Q}_{N+1}
$$

( $\bar{R}_{N}>0, \bar{Q}_{N+1}$ does not depend on $\varepsilon>0$ ). From the inequality (17) it follows that the series (4), obtained on constriction $\tau=\varphi(t, \varepsilon)$ is asymptotic for an exact solution $y(t, \varepsilon)$ of the problem (2) as $\varepsilon \rightarrow+0$. Theorem 4 is proved.

Example. Using the algorithm developed above, construct the main term of the asymptotic solution of the Cauchy problem:

$$
\varepsilon\left(\begin{array}{l}
y^{(1 / 2)} \\
z^{(1 / 2)}
\end{array}\right)=\left(\begin{array}{cc}
0 & 1 \\
-1 & 0
\end{array}\right)\left(\begin{array}{l}
y \\
z
\end{array}\right)+\left(\begin{array}{l}
h_{1}(t) \\
h_{2}(t)
\end{array}\right), \quad \begin{aligned}
& y(0, \varepsilon)=y^{0}, \\
& z(0, \varepsilon)=z^{0},
\end{aligned},
$$

where $t \in[0, T], T<1, \varepsilon>0$ small parameter. Eigen values of the matrix $A(t)$ of this system are numbers $\lambda_{1}(t) \equiv-i$, $\lambda_{2}(t) \equiv+i$. The corresponding eigenvectors $c_{j}(t)$ and eigenvectors $d_{j}(t)$ of the conjugate operator $A^{*}(t)$ have the form: 
Introduce regularizing variables:

$$
c_{1}=\left(\begin{array}{l}
-i \\
-1
\end{array}\right), \quad c_{2}=\left(\begin{array}{c}
i \\
-1
\end{array}\right), \quad d_{1}=\left(\begin{array}{l}
i \\
1
\end{array}\right), \quad d_{2}=\left(\begin{array}{c}
-i \\
1
\end{array}\right) .
$$

$$
\tau_{1}=-\frac{2 i}{\varepsilon} \sqrt{t} \equiv \varphi_{1}(t, \varepsilon), \quad \tau_{2}=\frac{2 i}{\varepsilon} \sqrt{t} \equiv \varphi_{1}(t, \varepsilon) .
$$

For extended functions $\tilde{w} \equiv\{\tilde{y}(t, \tau, \varepsilon), \tilde{z}(t, \tau, \varepsilon)\}$ we obtain the following problem:

$$
\varepsilon \sqrt{t} \frac{\partial \tilde{w}}{\partial t}+\sum_{j=1}^{2} \lambda_{j} \frac{\partial \tilde{w}}{\partial \tau_{j}}-A \tilde{w}=h(t), \quad \tilde{w}(0,0, \varepsilon)=w^{0},
$$

where $\tilde{w}=\{\tilde{y}, \tilde{z}\}, h(t)=\left\{h_{1}(t), h_{2}(t)\right\}, w^{0}=\left\{y^{0}, z^{0}\right\}$.

Defining a solution of this problem in the form of series

$$
\tilde{w}(t, u, \varepsilon)=\sum_{k=0}^{\infty} \varepsilon^{k} w_{k}(t, u)
$$

we get the following iteration systems:

$$
\begin{array}{ll}
L_{0} w_{0}(t, \tau) \equiv \sum_{j=1}^{2} \lambda_{j} \frac{\partial w_{0}}{\partial \tau_{j}}-A w_{0}=h(t), \quad w_{0}(0,0)=w^{0} ; & \left(\varepsilon^{0}\right) \\
L_{0} w_{1}(t, \tau)=-\sqrt{t} \frac{\partial w_{0}}{\partial t}, & w_{1}(0,0)=0 ; \\
L_{0} w_{k}(t, \tau)=-\sqrt{t} \frac{\partial w_{k-1}}{\partial t}, & w_{k}(0,0)=0, \quad k \geq 1 .
\end{array}
$$

We look for a solution of the equation $\left(\varepsilon^{0}\right)$ in the form of the functions:

$$
w_{0}(t, \tau)=w_{1}^{(0)}(t) e^{\tau_{1}}+w_{2}^{(0)}(t) e^{\tau_{2}}+w_{0}^{(0)}(t) .
$$

Putting (20) into the equation (17), and equating coefficients at the same exponentials and the free terms, we get:

$$
\begin{aligned}
& {\left[\lambda_{1} I-A\right] w_{1}^{(0)}(t)=0,} \\
& {\left[\lambda_{2} I-A\right] w_{2}^{(0)}(t)=0,} \\
& -A w_{0}^{(0)}(t)=h(t) .
\end{aligned}
$$
functions.

From the system (22) we find $w_{0}^{(0)}(t)=-A^{-1} h(t)$. In the equations (20) and (21) $w_{1}^{(0)}(t), w_{2}^{(0)}(t)$ arbitrary

Thus, we have defined solution (19) of the system $\left(\varepsilon^{0}\right)$ in the following way:

$$
w_{0}(t, \tau)=\alpha_{1}^{(0)}(t) c_{1} e^{\tau_{1}}+\alpha_{2}^{(0)}(t) c_{2} e^{\tau_{2}}-A^{-1} h(t),
$$

where $\alpha_{k}^{(0)}(t), k=1,2$ arbitrary functions.

We subject (23) to the initial condition $w_{0}(0,0)=w^{0}$ :

$$
\left(\begin{array}{l}
y^{0} \\
z^{0}
\end{array}\right)=\alpha_{1}^{(0)}(0)\left(\begin{array}{l}
-i \\
-1
\end{array}\right)+\alpha_{2}^{(0)}(0)\left(\begin{array}{c}
i \\
-1
\end{array}\right)-\left(\begin{array}{cc}
0 & -1 \\
1 & 0
\end{array}\right)\left(\begin{array}{l}
h_{1}(0) \\
h_{2}(0)
\end{array}\right),
$$

or

$$
\left\{\begin{array}{c}
-i \alpha_{1}^{(0)}(0)+i \alpha_{2}^{(0)}(0)+h_{2}(0)=y^{0} \\
-\alpha_{1}^{(0)}(0)-\alpha_{2}^{(0)}(0)-h_{1}(0)=z^{0}
\end{array}\right.
$$

then we get:

$$
\alpha_{1}^{(0)}(0)=\frac{z^{0}-h_{1}(0)-i\left[h_{2}(0)-y^{0}\right]}{2}, \quad \alpha_{2}^{(0)}(0)=\frac{z^{0}+h_{1}(0)+i\left[h_{2}(0)-y^{0}\right]}{2} .
$$

To uniquely define arbitrary functions $\alpha_{k}^{(0)}(t), k=1,2$, that are present in the solution (23) of the problem $\left(\varepsilon^{0}\right)$, we proceed to the next iteration problem $\left(\varepsilon^{1}\right)$.

First we calculate:

$$
\frac{\partial w_{0}(t, \tau)}{\partial t}=\dot{\alpha}_{1}^{(0)}(t) c_{1} e^{\tau_{1}}+\dot{\alpha}_{2}^{(0)}(t) c_{2} e^{\tau_{2}}-A^{-1} \dot{h}(t)
$$

Solution of the equation $\left(\varepsilon^{1}\right)$ is sought as a function: 


$$
w_{1}(t, \tau)=w_{1}^{(1)}(t) e^{\tau_{1}}+w_{2}^{(1)}(t) e^{\tau_{2}}+w_{0}^{(1)}(t) .
$$

Substituting (26) into the equation ( $\left.\varepsilon^{1}\right)$ (taking into account (25)), and equating coefficients at the same exponentials and the free terms, we have:

$$
\begin{gathered}
{\left[\lambda_{1} I-A\right] w_{1}^{(1)}(t)=-\sqrt{t} \dot{\alpha}_{1}^{(0)}(t),} \\
{\left[\lambda_{2} I-A\right] w_{2}^{(1)}(t)=-\sqrt{t} \dot{\alpha}_{2}^{(0)}(t),} \\
-A w_{0}^{(1)}(t)=-\sqrt{t} A^{-1} \dot{h}(t) .
\end{gathered}
$$

For solvability of the first two systems it is necessary and sufficient that $\dot{\alpha}_{k}^{(0)}(t)=0, k=1,2$. Taking into account the initial conditions $((24)$, we find the functions

$$
\alpha_{1}^{(0)}(t)=\alpha_{1}^{(0)}(0) \equiv \frac{z^{0}-h_{1}(0)-i\left[h_{2}(0)-y^{0}\right]}{2}, \quad \alpha_{2}^{(0)}(t)=\alpha_{2}^{(0)}(0) \equiv \frac{z^{0}+h_{1}(0)+i\left[h_{2}(0)-y^{0}\right]}{2},
$$

unambiguously.

Thus, we defined arbitrary functions $\alpha_{k}^{(0)}(t)=0, k=1,2$, in the solution (23), and thereby, uniquely determined the function (19) of the iteration problem $\left(\varepsilon^{0}\right)$, i.e., built the main term of the asymptotics of solutions to the problem (18):

$$
\left(\begin{array}{c}
y_{\varepsilon 0}(t) \\
z_{\varepsilon 0}(t)
\end{array}\right)=\left[\frac{z^{0}-h_{1}(0)-i\left(h_{2}(0)-y^{0}\right)}{2}\right]\left(\begin{array}{c}
-i \\
-1
\end{array}\right) e^{-\frac{2 i}{\varepsilon} \sqrt{t}}+\left[\frac{z^{0}+h_{1}(0)+i\left(h_{2}(0)-y^{0}\right)}{2}\right]\left(\begin{array}{c}
i \\
-1
\end{array}\right) e^{\frac{2 i}{\varepsilon} \sqrt{t}}-\left(\begin{array}{cc}
0 & -1 \\
1 & 0
\end{array}\right)\left(\begin{array}{c}
h_{1}(t) \\
h_{2}(t)
\end{array}\right)
$$

Acknowledgments. This work is supported by the grant AP05133858 "Contrast structures in singularly perturbed equations and their application in the theory of phase transitions" by the Scientific Committee of the Ministry of Education and Science of the Republic of Kazakhstan.

\section{REFERENCES}

[1] Kalimbetov, B.T. and Safonov, V.F. (1995) A regularization method for systems with unstable spectral value of the kernel of the integral operator. Journal Differential equations, 31, 647-656.

[2] Kalimbetov, B.T., Temirbekov, M.A. and Khabibullayev, Zh.O. (2012) Asymptotic solutions of singular perturbed problems with an instable spectrum of the limiting operator. Journal Abstract and Applied Analysis, 120192.

[3] Katugampola, U. (2015) Correction to "What is a fractional derivative?" by Ortigueira and Machado. Journal Computational Physics, 293, 4-13.

[4] Khalil, R., Al Horani, M., Yousef, A. and Sababheh, M. A new definition of fractional derivative. Journal Comput. Appl. Math., 264, 65-70.

[5] Khalil, R., Anderson, D. and Al Horani, M. (2014) Undetermined coefficients for local fractional differential equations. URL: https://www.researchgate.net/publication/303903312.

[6] Lomov, S.A. Introduction to General Theory of Singular Perturbations, 112, American Mathematical Society, Providence, USA. (1992) 